\title{
Recycling of Sintered Nd-Fe-B Magnets Doped with PrNd Nanoparticles
}

\author{
Xuefeng Zhang ${ }^{1,2 *}$, Fei Liu ${ }^{1,2}$, Yanli Liu ${ }^{1,2}$, Qiang Ma ${ }^{1,2}$, Yongfeng $\mathrm{Li}^{1,2}$, Qian Zhao ${ }^{1,2}$, \\ Gaofeng Wang' ${ }^{2}$, and Zhubai $\mathrm{Li}^{2}$ \\ ${ }^{1}$ School of Mathematics, Physics and Biological Engineering, Inner Mongolia University of Science \& Technology, Baotou 014010, China \\ ${ }^{2}$ Key Laboratory of Integrated Exploitation of Bayan Obo Multi-Metal Resources, Inner Mongolia University of Science \& Technology, \\ Baotou 014010, China
}

(Received 16 December 2014, Received in final form 29 March 2015, Accepted 13 April 2015)

\begin{abstract}
The waste of sintered Nd-Fe-B magnets was recycled using the method of dopingPrNd nanoparticles. The effect of PrNd nanoparticle doping on the magnetic properties of the regenerated magnets has been studied. As the content of the PrNd nanoparticles increases, the coercivity increases monotonically, whereas both the remanence and the maximum energy products reach the maximum values for 4 wt\% PrNd doping. Microstructural observation reveals that the appropriate addition of PrNd nanoparticles improves the magnetic properties and refines the grain. Domain investigation shows that the self-pinning effect of the rare earth (Re)-rich phase is enhanced by PrNd nano-particle doping. Compared to the magnet with $4 \mathrm{wt} \%$ PrNd alloy prepared using the dual-alloy method, the regenerated magnet doped with the same number of PrNd nanoparticles exhibits better magnetic properties and a more homogeneous microstructure. Therefore, it is concluded that PrNd nanoparticle doping is an efficient method for recycling the leftover scraps of Nd-Fe-B magnets.
\end{abstract}

Keywords : recycle, leftover scraps, Nd-Fe-B, PrNd nano-particle, magnetic properties

\section{Introduction}

Sintered Nd-Fe-B permanent magnets have been widely used due to their excellent magnetic properties [1-3]. The increasing demand has resulted in the enormous production of $\mathrm{Nd}-\mathrm{Fe}-\mathrm{B}$ magnets. At the same time, a great deal of waste is produced in the processes of industrial production, which include breaking raw materials, milling, pressing, and machining processes.It is necessary to recycle the waste and decrease the manufacturing cost.Therefore, studying the recycling of $\mathrm{Nd}-\mathrm{Fe}-\mathrm{B}$ magnet scrap is of considerable practical significance [4].

The aim of previous recycling processes was to purify the rare earth elements via chemical method for subsequent re-utilization [5]. However, the recovery ratio of this method is very low. The regenerated magnet can be prepared by milling the alloy particles of $\mathrm{Nd}-\mathrm{Fe}-\mathrm{B}$ waste which are used widely in factories, even though its magnetic properties are much inferior to those of conventional magnets. How can the recovery efficiency be improved

(C)The Korean Magnetics Society. All rights reserved.

*Corresponding author: Tel: +86-13190691928

Fax: +86-472-5954358, e-mail: xuefeng367@163.com and the loss of magnetic properties reduced? Many works have been attempted to increase the magnetic properties, such as by adding pure elements of $\mathrm{Dy}, \mathrm{Cu}$, and $\mathrm{Al}[6,7]$. Recently, it has been found that the magnetic properties, especially the coercivity, were enhanced greatly by doping nano-powder in the boundary of the $\mathrm{Nd}_{2} \mathrm{Fe}_{14} \mathrm{~B}$ matrix grains $[8,9]$. The recovery procedure is always accompanied by some Re-rich phase loss [10]. In work by Chao. Li et al., sintered magnets were recycled by doping $\mathrm{NdH}$ nanoparticles [11]. In this work, we concentrate on the method of doping PrNd nanoparticles in order to recycle left over scraps of sintered Nd-Fe-B magnets, and a comparison of magnetic properties is made between the magnets prepared by mixing PrNd nano-powder and by using the dual-alloy method.

\section{Experimental Procedure}

2.1. Regenerated magnet prepared by doping PrNd nano-particles in the boundary of matrix grains

Leftover scraps of sintered Nd-Fe-B magnets were used as starting materials. The nickel on the surface was removed from the selected magnets. Mechanical sanding and alcohol cleaning were applied to remove the oxide 
layer. Subsequently, hydrogen decrepitating at $150^{\circ} \mathrm{C}$ for $3 \mathrm{~h}$ [12-14] and ball milling for $45 \mathrm{~min}$ in petroleum ether were carried out to obtain fine Nd-Fe-B powders with an average size of $4.0 \mu \mathrm{m}$. On the other hand, PrNd nanoparticles with a composition of $\operatorname{Pr}_{25.5} \mathrm{Nd}_{74.5}$ (wt\%) were fabricated using the inert gas condensation method.The doping quantities of PrNd nanoparticles in the Nd-Fe-B powders were set to be $0 \mathrm{wt} \%, 2 \mathrm{wt} \%, 4 \mathrm{wt} \%, 6 \mathrm{wt} \%, 8$ $\mathrm{wt} \%$, and $10 \mathrm{wt} \%$. The two powders were mixed using high-energy ball milling without a medium. The mixed powder was then pressed under a pressure of $30 \mathrm{MPa}$ after alignment in a pulse magnetic field of $3 \mathrm{~T}$. The vacuum sintering was carried out at $1160^{\circ} \mathrm{C}$ for $2 \mathrm{~h}$. The magnets were annealed at $930^{\circ} \mathrm{C}$ for $110 \mathrm{~min}$ and subsequently at $620^{\circ} \mathrm{C}$ for $110 \mathrm{~min}$. The densities of magnets were measured using the Archimedes principle. The magnetic properties were examined using a NIM200C hysteresis graph. The phases, microstructure, and morphology of the sintered magnets were investigated using an x-ray diffraction (XRD) spectrum, scanning electron microscope (SEM), and dynamic domain observation instrument (Kerr microscope), respectively.

\subsection{Regenerated magnet prepared using the dual- alloy method}

The same amount of PrNd alloy as that of the PrNd nanoparticles was doped in Nd-Fe-B alloy. The two alloys were crushed and melted under an argon atmosphere in a vacuum induction furnace [15]. The ingots were mechanically pulverized into powders with a grain size of 3-5 $\mu \mathrm{m}$ using a pan grinder and a ball mill. Magnetic alignment was carried out in a pulsed field of $3 \mathrm{~T}$, and the mold was then pressed at a pressure of $30 \mathrm{MPa}$. The sintering process and post annealing were performed in the same way as that for the magnets doped with PrNd nanoparticles.

\section{Results and Discussion}

Fig. 1 shows the scanning electron microscope (SEM) image of PrNd nanoparticles. Most of the PrNd nanoparticles are about $50 \mathrm{~nm}$ in diameter.

Fig. 2 shows the magnetic properties of regenerated magnets as a function of $\mathrm{PrNd}$ nanoparticles content. The magnet without $\mathrm{PrNd}$ nanopowders show very low remanence $\left(B_{\mathrm{r}}=4.213 \mathrm{kGs}\right)$, coercivity $\left(H_{\mathrm{cj}}=0.152 \mathrm{kOe}\right)$, and maximum energy product $\left((B H)_{\max }=0.192 \mathrm{MGsOe}\right)$. $B_{\mathrm{r}},(B H)_{\max }$ and demagnetization curve rectangularity $(Q)$ increase with the increase in the number of PrNd nanoparticles, and achieve maxima of $11.34 \mathrm{kGs}, 31.16 \mathrm{MGsOe}$, and 0.951 for the magnet with $4 \mathrm{wt} \% \operatorname{PrNd}$ doping. With increasing the PrNd nano-powder, the $H_{c j}$ increases, but

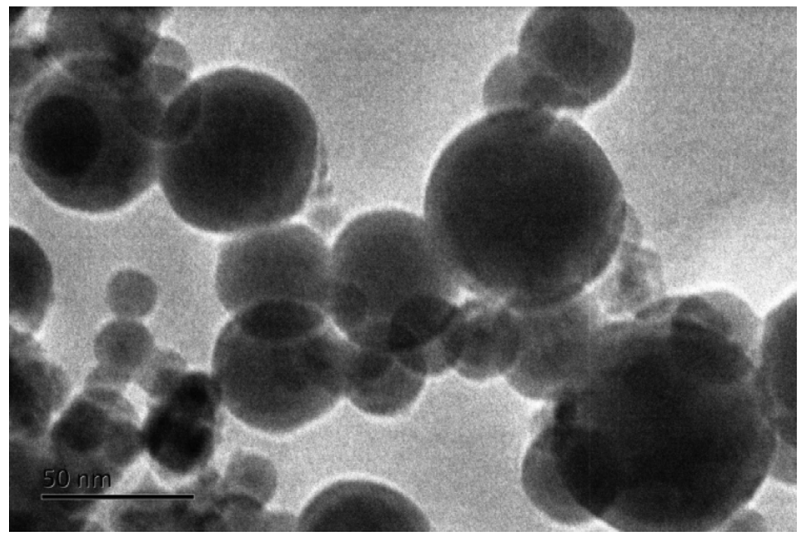

Fig. 1. SEM image of PrNd nanoparticles.

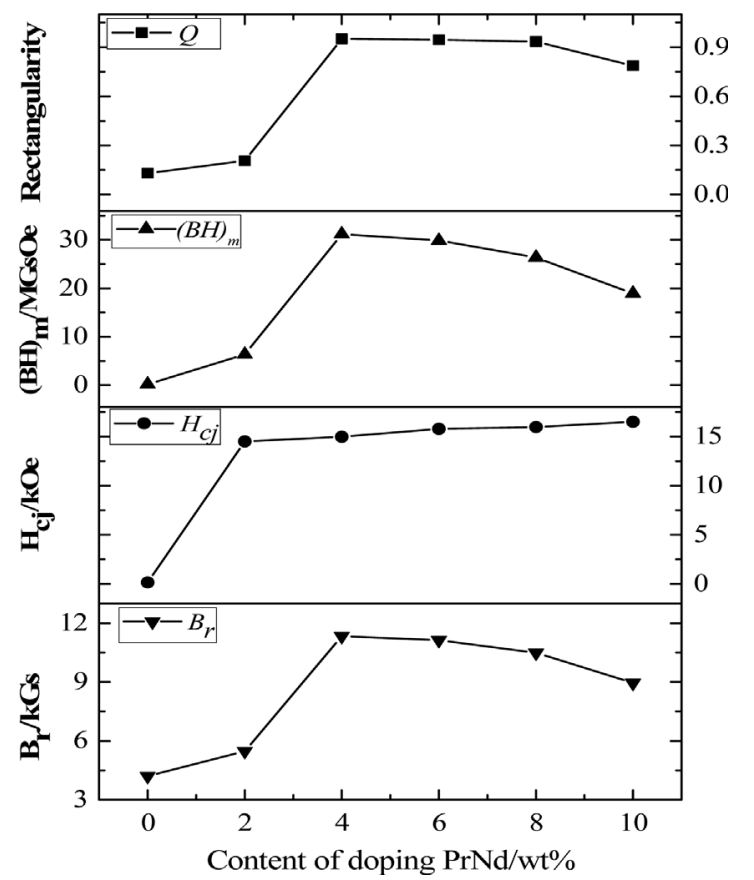

Fig. 2. Magnetic properties of regenerated magnets as a function of PrNd nanoparticle content.

the increment becomes slow when the number of nanoparticles is higher than $4 \mathrm{wt} \%$.

The densities of the regenerated magnets are shown in Fig. 3. It can be seen that the density reaches a maximum of $7.406 \mathrm{~g} / \mathrm{cm}^{3}$ for $4 \mathrm{wt} \%$ PrNd nanoparticle doping. The compactness of the samples deteriorates gradually when the nano-powder doping is higher than $4 \mathrm{wt} \%$. The related factors of remanence are expressed as

$$
B_{r}=A(1-\beta) \overline{\cos \theta} \frac{d}{d_{0}} M_{s},
$$

where $A$ denotes the volume fraction of domains, $\theta$ is the angle between the magnetization and the c-axis, $M_{s}$ is 


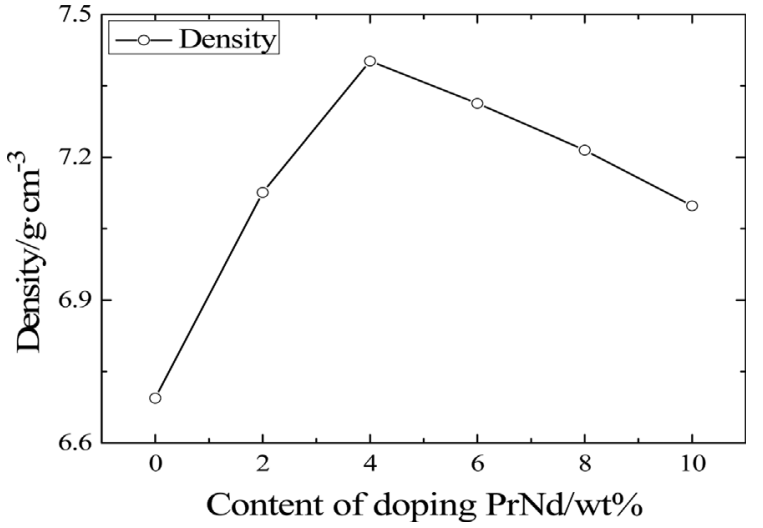

Fig. 3. Density of regenerated magnets with different $\mathrm{PrNd}$ nanoparticle contents.

spontaneous magnetization. $d / d_{0}$ is the opposite relative density of magnets and $\beta$ is the volume fraction of the non-magnetic phase. $B_{r}$ is enhanced by improving the magnet density and properly reducing the volume of the Re-rich phase.

Fig. 4 shows XRD patterns of regenerated magnets with different PrNd nanoparticle contents. There is no new phase after doping of the PrNd nanoparticles, indicating that the composition of the regenerated magnets did not change, as shown in Fig. 4. It can be seen that the intensity of the diffraction peak of $\operatorname{Pr}_{2} \mathrm{O}_{3}$ and $\mathrm{Nd}_{2} \mathrm{O}_{3}$ becomes stronger with the increase of the nano-powder content, indicating that the volume of the Re-rich phase increases. In addition, the diffraction peaks of the $\mathrm{Nd}_{2} \mathrm{Fe}_{14} \mathrm{~B}$ phase are broadened for the nano-powder doping, and the peak width changes insignificantly for a $\mathrm{PrNd}$ nanoparticles content higher than $4 \mathrm{wt} \%$. This result could indicate the grain refinement due to the addition of PrNd nanoparticles.

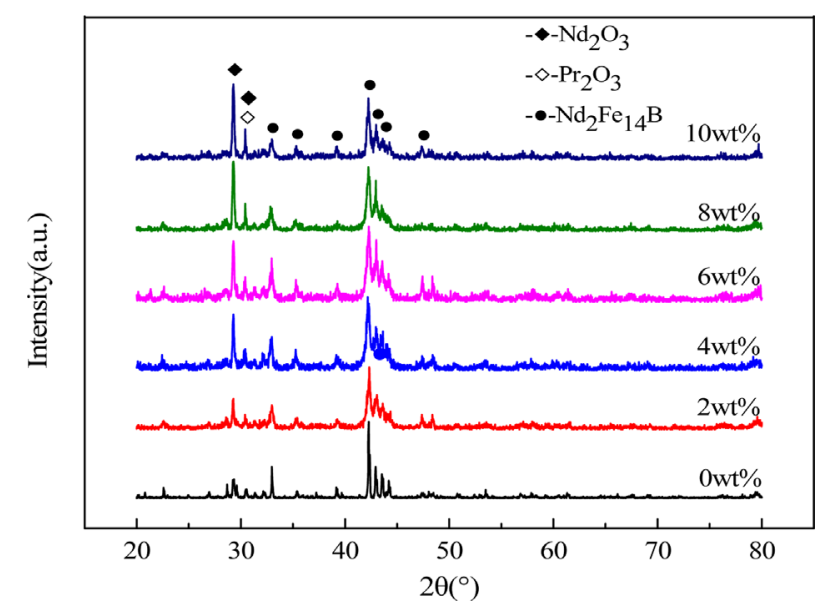

Fig. 4. (Color online) XRD patterns of regenerated magnets with different PrNd nanoparticle contents.
SEM images of regenerated magnets are shown in Fig. 5. It can be seen that the microstructure of the magnet without PrNd nano-powders is inhomogeneous, and the grains are large. The major reason for the low coercivity is the abnormal grain growth caused by the absence of Re-rich phase in the recovery procedure. The grain size becomes smaller with the addition of PrNd nano-powders, which is responsible for the enhanced coercivity. In addition, a small amount of Re-rich phase is observed in Fig. 5(a), which attaches to the surface of the $\mathrm{Nd}_{2} \mathrm{Fe}_{14} \mathrm{~B}$ grains, taking the form of the particle phase. With increasing PrNd nanoparticles content, the Nd-Fe-B grains are wrapped in a thin layer of the Re-rich phase (shown in Fig. 5(c)). The Re-rich phase is mostly enriched at the grain boundary which weakens the coupling between the hard phase grains. The abnormal grain growth is restrained favorably when the Re-rich phase is formed in an appropriate amount. Furthermore, the enriched Re-rich phase at grain boundaries provides a mass transport pass for the growth of oriented Nd-Fe-B grains with the easy magnetization axis paralleling the direction of magnetic alignment. This leads to the enhancement of coercivity in the PrNd nanoparticles doped magnet. An obvious agglomeration phenomenon is observed in the Re-rich phase for $8 \mathrm{wt} \%$ and $10 \mathrm{wt} \%$ PrNd nanoparticles doped magnets as shown in Fig. 5(e) and Fig. 5(f). The agglomeration of the Re-rich phase, which results from the excessively doped PrNd nanoparticles, reduces the compactness of the sintered regenerated magnets and causes the decrease of remanence.

In order to further investigate the mechanism of coercivity enhancement, the Kerr microscope was used for domain observation in high magnetic fields [16-18]. The change of microstructure with PrNd nanoparticles addition is also realized from the microscope image in Fig. 6, which is accordant with that observed by SEM. Dark and bright domains are observed in the same grain, indicating that the direction of magnetization is parallel or antiparallel to the c-axis. In Fig. 6(a), many continuous domains through the adjacent grains are observed in the regenerated magnet without PrNd nano-powder, which is not a local phenomenon (shown in Fig. 6(a)). One of the reasons for this phenomenon is the Re-rich phase loss, which should be responsible for the poor magnetic properties. In Fig. 6(b), for the doping $4 \mathrm{wt} \% \mathrm{PrNd}$ nanoparticles, there are rarely continuous domains in the magnet, which is consistent with the self-pinning mechanism of Zhao et al. [19, 20].

According to Zhao et al. [19], the magnetic reversal in nanostructured permanent magnets is normally composed of three steps: 1. nucleation of the domain wall at the 

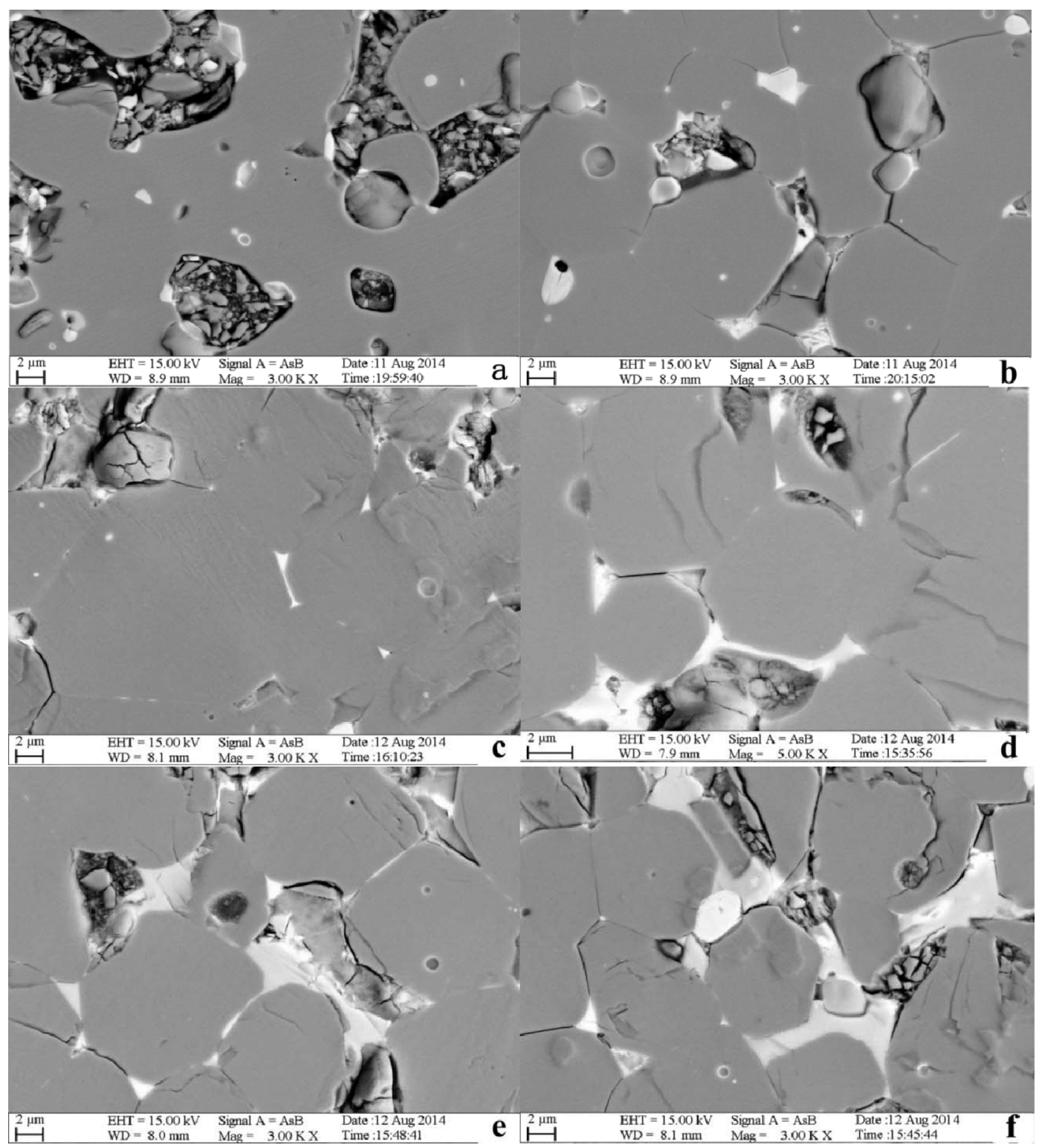

Fig. 5. SEM patterns of regenerated magnets for different PrNd nanoparticles contents: (a) 0 wt $\%$, (b) 2 wt $\%$, (c) 4 wt $\%$, (d) 6 $w t \%$, (e) $8 w t \%$, and (f) $10 w t \%$.

defects where the crystalline anisotropy is locally small, 2. the evolution of the domain wall near the nucleation center, and 3. the motion of the domain wall into the main phase [21]. The coercivity is given by the pinning field which hinders the motion of the domain wall. Without the addition of the PrNd nanoparticles, the domain walls can move freely in the main phase between different grains as can be seen in Fig. 6(a), which decreases the coercivity significantly. The addition of the PrNd nanoparticles at the grain boundaries enhances the self-pinning field considerably, so that the domain wall is limited in the grain with the defect (Fig. 6(b)). As a result, the domain wall is restrained near the nucleation center in the demagnetization process and the self-pinning field and the coercivity can be increased significantly.

Other popular coercivity models in permanent magnets are the nucleation and pinning models [22,23]. According to the nucleation model, the coercivity is given by:

$$
\mu_{0} H_{c}=\frac{K}{M_{s}} \alpha-N_{e f f} \mu_{0} M_{s}
$$

where the microstructural parameter, $\alpha$, describes the reduction of the ideal nucleation field due to the microstructure. $K$ is the magnetocrystalline anisotropy constant. The effective demagnetization factor, $N_{\text {eff, }}$, takes into account the enhanced local stray fields at the edges and 


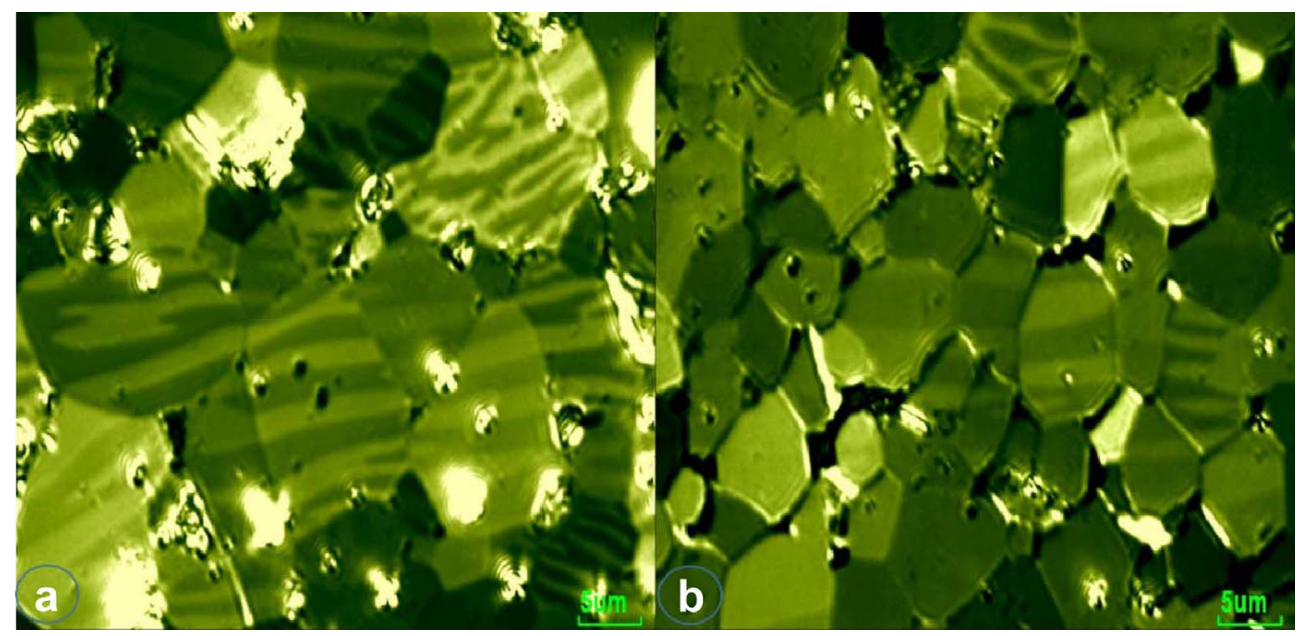

Fig. 6. (Color online) Domain patterns of regenerated magnets with different PrNd nanoparticles contents: (a) 0 wt $\%$ and (b) 4 wt $\%$.

corners of the polyhedral grains. With the proper adoption of the parameters, the nucleation model can yield values of the coercivity comparable to those shown in Fig. 2. However, it is difficult to explain the enhancement of the coercivity due to the addition of the PrNd particles in the present work based on the nucleation model, which is basically a pinning mechanism. The traditional pinning model, on the other hand, does not provide the origin of the domain wall (which is the nucleation at the defects based on the self-pinning model), so that it cannot explain the coercivity mechanism completely [24].

Compared with the regenerated magnet with 4 wt $\%$ PrNd nanoparticles, the magnet prepared with the dualalloy method with $4 \mathrm{wt} \%$ PrNd alloy shows relatively weaker magnetic properties $\left(B_{\mathrm{r}}=10.35 \mathrm{kGs}, H_{\mathrm{cj}}=11.96\right.$ $\left.\mathrm{kOe},(B H)_{\max }=25.16 \mathrm{MGsOe}\right)$. The microstructure of the magnet with $4 \mathrm{wt} \%$ PrNd alloy is shown in Fig. 7. Even though the Re-rich phase is more inhomogeneous than

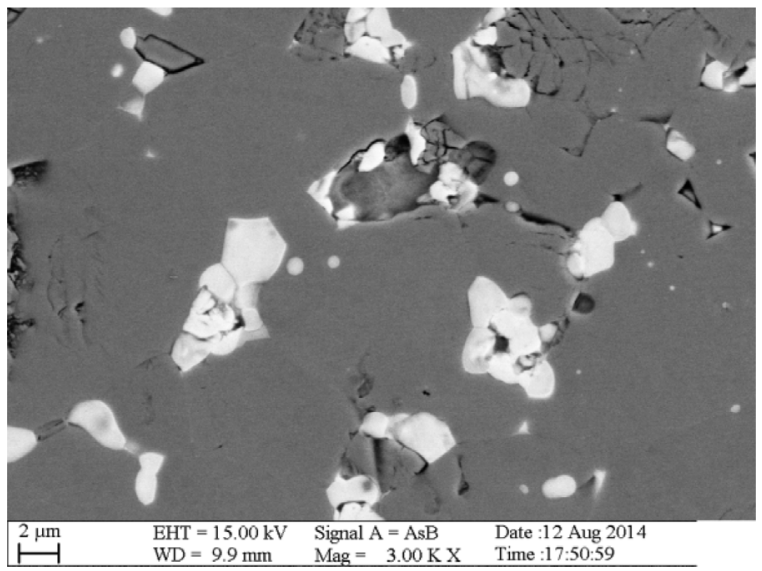

Fig. 7. SEM image of the regenerated magnet with $4 \mathrm{wt} \%$ PrNd alloy. that of the magnet with $4 \mathrm{wt} \%$ PrNd nanoparticles shown in Fig. 5(c), the agglomeration of the Re-rich phase is observed, which is the reason for the worse magnetic properties in the magnet prepared using the dual-alloy method.

\section{Conclusions}

Leftover scraps of Nd-Fe-B sintered magnets were recycled using the method of doping PrNd nanoparticles. As the PrNd nanoparticles content increases, the $H_{c j}$ is enhanced progressively, while $B_{r}$ and $(B H)_{\max }$ achieve maximum values for $4 \mathrm{wt} \%$ PrNd doping. Microstructure investigation shows that for the doping of $4 \mathrm{wt} \% \mathrm{PrNd}$ nanoparticles, the Re-rich phase is mainly enriched at grain boundaries, which is beneficial to the compactness of the regenerated magnet and the microstructure homogenization. The uniform distribution of the Re-rich phase has the self-pinning effect at grain boundaries, there by improving the coercivity of the regenerated magnets. Compared with the magnet prepared using the dual-alloy method with $4 \mathrm{wt} \%$ PrNd alloy, the regenerated magnet with $4 \mathrm{wt} \%$ PrNd nanoparticles shows better magnetic properties and a more homogeneous microstructure. Finally, PrNd nanoparticles doping is a valuable method for recycling the waste of Nd-Fe-B magnets.

\section{Acknowledgements}

This paper was supported by the Inner Mongolia Innovative Research Team (3400102), Provincial Major Science and Technology Innovation Project of Inner Mongolia (2009J1006), Baotou Key Project of Rare Earth (2012R1006), Inner Mongolia Science Foundation 
(2012MS0802), Science and Technology Innovation Project of Inner Mongolia University of Science and Technology (2012NCL048).

\section{References}

[1] M. Sagawa, S. Fujimura, N. Togawa, H. Yamamoto, and Y. Matsuura, J. Appl. Phys. 55, 2083 (1984).

[2] J. J. Croat, J. F. Herbst, R. W. Lee, and F. E. Pinkerton, J. Appl. Phys. 55, 2078 (1984).

[3] S. Sugimoto, J. Phys. D: Appl. Phys. 44, 0cc64001 (2011).

[4] K. Binnemans, P. T. Jones, B. Blanpain, T. V. Gerven, Y. X. Yang, A. Walton, and M. Buchert, J. Clean. Prod. 51, 1 (2013).

[5] T. Horikawa, K. Miura, M. Itoh, and Ken-Ichi Machida, J. Alloy. Compd. 408, 1386 (2006).

[6] N. C. Koon, B. N. Das, M. Rubinstein, and J. Tyson, J. Appl. Phys. 57, 4091 (1985).

[7] L. G. Pampillo, F. D. Saccone, and H. R. M. Sirkin, Physica B 389, 172 (2007).

[8] W. Q. Liu, H. Sun, X. F. Yi, X. C. Liu, D. T. Zhang, M. Yuea, and J. X. Zhang, J. Alloy. Compd. 501, 67 (2010).

[9] C. Sun, W. Q. Liu, H. Sun, M. Yue, X. F. Yi, and J. W. Chen, J. Mater. Sci. Technol. 28, 927 (2012).

[10] M. Zakotnik, I. R. Harris, and A. J. Williams, J. Alloy. Compd. 469, 314 (2009).

[11] C. Li, W. Q. Liu, M. Yue, D. T. Zhang, and J. X. Zhang,
Funct. Mater. 45, 17059 (2014). (in chinese)

[12] S. Liesert, D. Fruchart, P. de Rango, S. Rivoirard, J. L. Soubeyroux,R. Perrier de la Bfithie, and R. Tournier, J. Alloy. Compd. 262, 366 (1997).

[13] M. Zakotnik, I. R. Harris, and A. J. Williams, J. Alloy. Compd. 450, 525 (2008).

[14] H. W. Kwon, D. H. Kim, and J. H. Yu, J. Magn. 15, 155 (2010).

[15] J. Zhou, W. Song, and X. D. Pei, Met. Funct. Mater. 18, 5 (2011). (in chinese)

[16] M. Takezawa, Y. Kimura, Y. Morimoto, and J. Yamasaki, IEEE Trans. Magn. 49, 3262 (2013).

[17] M. Takezawa, Y. Kimura, Y. Morimoto, and J. Yamasaki, IEEE Trans. Magn. 47, 3256 (2011).

[18] J. Thielsch, D. Hinz, L. Schultz, and O. Gutfleisch, J. Magn. Magn. Mater. 322, 3208 (2010).

[19] G. P. Zhao, X. L. Wang, C. Yang, L. H. Xie, and G. Zhou, J. Appl. Phys. 101, 09K102 (2007).

[20] G. P. Zhao, H. W. Zhang, Y. P. Feng, Chun Yang, and C. W. Huang, Comp. Mater. Sci. 44, 122 (2008).

[21] G. P. Zhao, M. G. Zhao, H. S. Lim, Y. P. Feng, and C. K. Ong, Appl. Phys. Lett. 87, 162513 (2005).

[22] H. Knomüller and D. Goll, Scripta Mater. 47, 511 (2002).

[23] H. Knomüller and D. Goll, Scripta Mater. 47, 545 (2002).

[24] G. P. Zhao, F. Morvan, and X. L. Wan, Rev. Nanosci. Nanotechnol. 3, 227 (2014). 